\title{
de Sitter symmetry of Neveu-Schwarz spinors
}

\author{
Henri Epstein ${ }^{a}$ and Ugo Moschella ${ }^{b, c}$ \\ ${ }^{a}$ Institut des Hautes Etudes Scientifiques (IHES), \\ 35, Route de Chartres, 91440 Bures-sur-Yvette, France \\ ${ }^{b}$ Università dell'Insubria, DiSat, \\ Via Valleggio 11, 21100, Como, Italia \\ ${ }^{c}$ INFN sezione di Milano, \\ Via Celoria 16, 20133 Milano, Italia \\ E-mail: epstein@hes.fr, ugo.moschella@uninsubria.it
}

Abstract: We study the relations between Dirac fields living on the 2-dimensional Lorentzian cylinder and the ones living on the double-covering of the 2-dimensional de Sitter manifold, here identified as a certain coset space of the group $\mathrm{SL}(2, R)$. We show that there is an extended notion of de Sitter covariance only for Dirac fields having the Neveu-Schwarz anti-periodicity and construct the relevant cocycle. Finally, we show that the de Sitter symmetry is naturally inherited by the Neveu-Schwarz massless Dirac field on the cylinder.

Keywords: Field Theories in Lower Dimensions, Space-Time Symmetries, Integrable Field Theories, 2D Gravity

ARXIV EPRINT: 1604.08385 


\section{Contents}

1 Introduction 1

2 The Dirac equation on the 2-dimensional Minkowskian cylinder $\quad 2$

2.1 Scalar ancestors 4

3 The Dirac equation on the two-dimensional de Sitter spacetime $\quad 5$

4 Another equation by Dirac $\quad 6$

$\begin{array}{lll}4.1 & \text { Spinorial plane waves } & 7\end{array}$

$\begin{array}{lll}5 & \text { Spin group and de Sitter covariance } & 10\end{array}$

6 The symmetric space $\operatorname{Sp}(1,2) / A$ as the double covering of the two$\begin{array}{ll}\text { dimensional de Sitter spacetime. } & 11\end{array}$

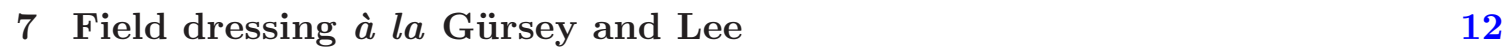

8 Cocyclic covariance of the de Sitter Dirac-Fock-Ivanenko field 14

9 Massless fields: from the the de Sitter manifold to the cylinder and back 15

$\begin{array}{ll}\text { A Derivation of the Gursey and Lee's dressing } & 17\end{array}$

$\begin{array}{ll}\text { B Maximally analytic vacua } & 18\end{array}$

\section{Introduction}

There are two spin structures and, correspondingly, two possible choices of free fermions on the (Minkowskian or Euclidean) cylinder. According to the terminology of string theory, fields which are periodic are called Ramond (R) fields, fields which are anti-periodic are called Neveu-Schwarz (NS) fields. The conformal mapping of the Euclidean cylinder onto the punctured complex plane changes this situation: here Neveu-Schwarz fields are periodic and Ramond fields are anti-periodic, showing that the NS condition is the more natural one, the one which fits better with conformal invariance (see e.g [1-3]).

In this paper we examine another kind of conformal mapping: the map of a portion of the Minkowskian cylinder onto the two-dimensional de Sitter spacetime and the transformation properties of quantum Dirac fields under such mapping. The fields obtained in this way on the two-dimensional de Sitter universe have no a priori reason to fit with the de Sitter symmetry of the manifold. We will show however that the canonical quantum Dirac field subject to the NS anti-periodicity condition does enjoy a certain natural form of de 
Sitter covariance. Also, the inverse image of that representation shows that NS canonical Dirac fields carry a hidden de Sitter symmetry already on the cylinder. On the other hand, the field which satisfies the Ramond periodicity condition transforms covariantly only under the (compact) subgroup of spatial rotations while the boosts are broken.

The construction goes as follows: after recalling the canonical quantizations of either periodic (R) or anti-periodic (NS) Dirac fields on the Minkowskian cylinder, we consider quantum Dirac fields on the two-dimensional de Sitter universe. There are two possible ways of writing a Dirac equation on that manifold. The first equation, recalled in section 3 , is the standard Dirac-Fock-Ivanenko equation constructed in terms of the spin connection (see e.g. [4]). The second equation, described in section 4, arises in a construction due to Dirac himself [5] that is directly related to a particular unitary representation of the de Sitter group [6]. In the four-dimensional case the relation between the two equations has been elucidated by F. Gürsey and T. D. Lee [7]. The two-dimensional case that we are considering here is more subtle for topological reasons. It turns out that a transformation matrix field à la Gürsey and Lee interpolating between the two Dirac fields only exists on the double covering of the (two-dimensional) de Sitter manifold - identified in section 6 as the coset space of the spin group $\operatorname{SL}(2, R)$ over the abelian subgroup $A$. This construction allows to transfer the representation of the de Sitter group naturally associated to the Dirac's equation of section 4 to the fields that are solution of the Dirac-Fock-Ivanenko equation. The de Sitter covariance is now expressed in terms of a cocycle that we explicitly construct in section 8. The result is that Neveu-Schwarz fields on the double covering of the de Sitter hyperboloid are fully de Sitter covariant while Ramond fields are only rotation invariant.

In the last section we exhibit the conformal transformation relating de Sitter covariant field on the de Sitter manifold and the massless NS Dirac field on the cylinder, which therefore carries a hidden de Sitter symmetry. On the other hand the same mapping applied to the Ramond field produces a field which only has the rotation invariance. It is well known that fields do not have to be single-valued in a non simply connected space [8-10]. Our result helps to understand the naturalness of the NS boundary condition in the study of 2-dim QFT models $[1,11,12]$.

The formalism and the results presented here may be useful in the study of various conformal field theories on the two-dimensional de Sitter universe. In an accompanying paper we will present a construction of the de Sitter -Thirring model.

\section{The Dirac equation on the 2-dimensional Minkowskian cylinder}

Consider a Minkowskian cylinder parametrised with a time coordinate $x^{0}=t$ and a periodic spatial coordinate $x^{1}=\theta$ (with $-\pi<\theta<\pi$ ); the metric coincides with the Minkowski metric:

$$
d s^{2}=d t^{2}-d \theta^{2}
$$

apart from the periodicity of the $\theta$ coordinate. The Clifford anti-commutation relations

$$
\begin{aligned}
\gamma^{a} \gamma^{b}+\gamma^{b} \gamma^{a} & =2 \eta^{a b}, \\
\eta_{a b}=\operatorname{diag}(1,-1), \quad a, b & =0,1,
\end{aligned}
$$


may be realised by the following choice of gamma matrices:

$$
\gamma^{0}=\left(\begin{array}{ll}
0 & 1 \\
1 & 0
\end{array}\right), \quad \gamma^{1}=\left(\begin{array}{cc}
0 & 1 \\
-1 & 0
\end{array}\right)
$$

There are two inequivalent spin structures on the cylinder which correspond to two different monodromies for the spinor fields: periodic spinors:

$$
\text { (R) } \quad \psi(t, \theta+2 \pi)=\psi(t, \theta)
$$

and anti-periodic spinors:

$$
(N S) \quad \psi(t, \theta+2 \pi)=-\psi(t, \theta) .
$$

Let us consider now the massless Dirac equation on the cylinder:

$$
i \gamma^{a} \partial_{a} \psi=0
$$

There are at least two canonical quantum fields that solve this equation and that correspond to the above two monodromies. To fix notations and for the reader's convenience we recall them here. They are constructed in terms of two sets of creation and annihilation operators

$$
a_{j}(p), b_{j}(p), a_{j}^{*}(p), b_{j}^{*}(p), \quad j=1,2, \quad p \in \mathbf{Z}, \quad p \geq 0,
$$

acting in a Fock space with vacuum $\Omega$

$$
a_{j}(p) \Omega=0, \quad b_{j}(p) \Omega=0,
$$

and obeying the canonical anti-commutation relations:

$$
\left\{a_{j}(p), a_{k}^{*}(q)\right\}=\delta_{j k} \delta_{p q}, \quad\left\{b_{j}(p), b_{k}^{*}(q)\right\}=\delta_{j k} \delta_{p q} .
$$

Every other anticommutator vanishes.

Ramond canonical field. In terms of the light-cone variables

$$
u=t+\theta, \quad v=t-\theta
$$

the components of the quantum Ramond-Dirac field are written as follows:

$$
\begin{aligned}
& \psi_{1}^{\mathrm{R}}(x)=\psi_{1}^{\mathrm{R}}(u)=\frac{1}{2 \sqrt{\pi}}\left(a_{1}^{*}(0)+b_{1}(0)\right)+\frac{1}{\sqrt{2 \pi}} \sum_{p>0}\left(a_{1}^{*}(p) e^{i p u}+b_{1}(p) e^{-i p u}\right), \\
& \psi_{2}^{\mathrm{R}}(x)=\psi_{2}^{\mathrm{R}}(v)=\frac{1}{2 \sqrt{\pi}}\left(a_{2}^{*}(0)+b_{2}(0)\right)+\frac{1}{\sqrt{2 \pi}} \sum_{p>0}\left(a_{2}^{*}(p) e^{i p v}+b_{2}(p) e^{-i p v}\right) .
\end{aligned}
$$

(There is however a certain arbitrariness in the choice of the canonical operators relative to the zero modes. We agree here to the standard choice). 
$\psi^{\mathrm{R}}$ is a univalued function on the cylinder. The nonvaninshing 2-pt functions are given by

$$
\begin{aligned}
& w_{1}(x, y)=\left(\Omega, \psi_{1}^{\mathrm{R}}(x) \psi_{1}^{\mathrm{R} *}(y) \Omega\right)=\frac{1}{2 \pi}\left[\frac{1}{2}+\sum_{p>0} e^{-i p\left(u-u^{\prime}\right)}\right]=-\frac{i}{4 \pi} \cot \left(\frac{u-u^{\prime}-i 0}{2}\right) \\
& w_{2}(x, y)=\left(\Omega, \psi_{2}^{\mathrm{R}}(x) \psi_{2}^{\mathrm{R} *}(y) \Omega\right)=\frac{1}{2 \pi}\left[\frac{1}{2}+\sum_{p>0} e^{-i p\left(v-v^{\prime}\right)}\right]=-\frac{i}{4 \pi} \cot \left(\frac{v-v^{\prime}-i 0}{2}\right)
\end{aligned}
$$

In the usual matrix form (with $\bar{\psi}=\psi^{+} \gamma^{0}$ )

$$
\left(\Omega, \psi^{\mathrm{R}}(x) \bar{\psi}^{\mathrm{R}}(y) \Omega\right)=-\frac{i}{4 \pi}\left(\begin{array}{cc}
0 & \cot \left(\frac{1}{2}\left(u-u^{\prime}\right)\right) \\
\cot \left(\frac{1}{2}\left(v-v^{\prime}\right)\right) & 0
\end{array}\right) .
$$

Neveu-Schwarz canonical field. Neveu-Schwarz Dirac fields are obtained by summing over half-integer momenta; one consequence of the shift $p \rightarrow p+1 / 2$ is that there are no more zero modes and that all the modes enter in the field expansion with the same normalization:

$$
\begin{aligned}
& \psi_{1}^{\mathrm{NS}}(u)=\frac{1}{\sqrt{2 \pi}} \sum_{p \geq 0}\left(a_{1}^{*}(p) e^{i p u+i u / 2}+b_{1}(p) e^{-i p u-i u / 2}\right) \\
& \psi_{2}^{\mathrm{NS}}(v)=\frac{1}{\sqrt{2 \pi}} \sum_{p \geq 0}\left(a_{2}^{*}(p) e^{i p v+i v / 2}+b_{2}(p) e^{-i p v-i v / 2}\right)
\end{aligned}
$$

Here $\psi^{\text {NS }}$ is a bivalued function on the cylinder. The 2-pt functions is now given by

$$
\left(\Omega, \psi^{\mathrm{NS}}(x) \bar{\psi}^{\mathrm{NS}}(y) \Omega\right)=-\frac{i}{4 \pi}\left(\begin{array}{cc}
0 & \frac{1}{\sin \left(\frac{1}{2}\left(u-u^{\prime}\right)\right)} \\
\frac{1}{\sin \left(\frac{1}{2}\left(v-v^{\prime}\right)\right)} & 0
\end{array}\right) .
$$

\subsection{Scalar ancestors}

In correspondence with the above construction one may also introduce two scalar massless fields. The field that one would naturally get by the method of canonical quantisation, defined on the cylinder itself, would be plagued by an infrared divergence which arises from a diverging normalisation of the zero mode. This divergence may be cared by replacing the (infinite) constant zero mode by a normalised zero mode growing with time Performing this regularisation one gets a canonical quantum field but the price to pay is that positivedefiniteness has been lost; the two point function is

$$
\begin{aligned}
\left(\Omega, \varphi^{\mathrm{R}}(x) \varphi^{\mathrm{R}}(y) \Omega\right)=\widehat{D}_{0}(x-y) & =-\frac{i x^{0}-i y^{0}}{4 \pi}+\frac{1}{4 \pi} \sum_{p \in \mathbf{Z} \backslash\{0\}} \frac{e^{-i|p|\left(x^{0}-y^{0}\right)+i p\left(x^{1}-y^{1}\right)}}{|p|} \\
& =-\frac{1}{4 \pi} \log \left(-\frac{1}{4} \sin \left(\frac{1}{2}\left(u-u^{\prime}\right)\right) \sin \left(\frac{1}{2}\left(v-v^{\prime}\right)\right)\right)
\end{aligned}
$$

so that

$$
\left(\Omega, \psi^{\mathrm{R}}(x) \bar{\psi}^{\mathrm{R}}(y) \Omega\right)=i \gamma^{a} \partial_{a} \widehat{D}_{0}(x-y)
$$


The two-point function of the second scalar field is constructed by shifting the series in eq. (2.15) to half integer momenta and suppressing the zero modes as follows:

$$
\begin{aligned}
\left(\Omega, \varphi^{\mathrm{NS}}(x) \varphi^{\mathrm{NS}}(y) \Omega\right)=D_{\mathrm{NS}}(x-y) & =\frac{1}{4 \pi} \sum_{p \in \mathbf{Z}} \frac{e^{-i|p+1 / 2|\left(x^{0}-y^{0}\right)+i(p+1 / 2)\left(x^{1}-y^{1}\right)}}{|p+1 / 2|} \\
& =\frac{1}{4 \pi} \log \left(-\cot \left(\frac{1}{4}\left(u-u^{\prime}\right)\right) \cot \left(\frac{1}{4}\left(v-v^{\prime}\right)\right)\right)
\end{aligned}
$$

so that

$$
\left(\Omega, \psi^{\mathrm{NS}}(x) \bar{\psi}^{\mathrm{NS}}(y) \Omega\right)=i \gamma^{a} \partial_{a} D_{\mathrm{NS}}(x-y) .
$$

\section{The Dirac equation on the two-dimensional de Sitter spacetime}

Consider now a (classical or quantum) massless Dirac spinor having the following form:

$$
\psi=(\cos t)^{-\frac{1}{2}} \phi
$$

The spinor $\phi$ itself solves a closely (conformally) related Dirac equation

$$
i \gamma^{a} \partial_{a} \phi+\frac{i}{2} \tan t \gamma^{0} \phi=0
$$

which may be interpreted as the massless Dirac (Fock-Ivanenko) equation on the de Sitter manifold. Let us consider indeed the two dimensional de Sitter spacetime represented as the one-sheeted hyperboloid

$$
d S_{2}=\left\{X \in M_{3}:\left(X^{0}\right)^{2}-\left(X^{1}\right)^{2}-\left(X^{2}\right)^{2}=-1\right\}
$$

embedded in the three-dimensional Minkowski spacetime $M_{3}$ whose metric tensor is

$$
\eta_{\alpha \beta}=\operatorname{diag}(1,-1,-1) \quad \alpha, \beta=0,1,2 .
$$

The following choice of coordinates

$$
X(t, \theta)=\left\{\begin{array}{l}
X^{0}=r \tan t \\
X^{1}=r \sin \theta / \cos t \\
X^{2}=r \cos \theta / \cos t
\end{array}\right.
$$

covers the complement of the lightcone in the ambient space: all the events which are spacelike w.r.t. to the origin $(0,0,1)$. In the above coordinates the de Sitter manifold $(r=R=1)$ is manifestly conformal to a portion (i.e. $-\pi / 2<t<\pi / 2)$ of the Minkowskian cylinder:

$$
d s^{2}=\frac{1}{\cos ^{2} t}\left(d t^{2}-d \theta^{2}\right)
$$

the interval of the ambient spacetime (restricted to $r=1$ ) is given by

$$
(X-Y)^{2}=\frac{2 \cos \left(\theta-\theta^{\prime}\right)-2 \cos \left(t-t^{\prime}\right)}{\cos t \cos t^{\prime}} .
$$


The components of the natural zweibein $e_{a}^{i}$ are

$$
e_{0}^{t}=\cos t, \quad e_{1}^{t}=0, \quad e_{0}^{\theta}=0, \quad e_{1}^{\theta}=\cos t,
$$

and the curved-space matrices $\alpha^{i}=e_{a}^{i} \gamma^{a}$ are simply proportional to the flat space gamma matrices:

$$
\alpha^{t}=\left(\begin{array}{cc}
0 & \cos t \\
\cos t & 0
\end{array}\right)=(\cos t) \gamma^{0}, \quad \alpha^{\theta}=\left(\begin{array}{cc}
0 & \cos t \\
-\cos t & 0
\end{array}\right)=(\cos t) \gamma^{1} .
$$

In two-dimensions there is only one nonvanishing component of the spin connection $\omega_{\mu a b}=$ $e_{a}^{\nu} \nabla_{\mu} e_{b \nu}$. In our example it is given by $\omega_{\theta 01}=-\omega_{\theta 10}=\tan t$. Correspondingly

$$
\Gamma_{t}=0, \quad \Gamma_{\theta}=\frac{1}{4}\left[\gamma^{0}, \gamma^{1}\right] \omega_{\theta 01}=\tan t\left(\begin{array}{cc}
-\frac{1}{2} & 0 \\
0 & \frac{1}{2}
\end{array}\right) .
$$

Putting everything together, the massless Dirac equation on the de Sitter manifold is finally written as follows:

$$
i \alpha^{i}\left(\partial_{i}+\Gamma_{i}\right) \phi=i \alpha^{i} \partial_{i} \phi+i \alpha^{\theta} \Gamma_{\theta} \phi=i \cos t\left(\gamma^{a} \partial_{a} \phi+\frac{i}{2} \tan t \gamma^{0} \phi\right)=0
$$

Eq. (3.11) indeed coincides with eq. (3.2). In the general case a mass term has to be added

$$
i \alpha^{i}\left(\partial_{i}+\Gamma_{i}\right) \phi-m \phi=0 .
$$

and this of course breaks the conformal invariance.

\section{Another equation by Dirac}

In this section we elaborate on another first order equation for a spinor on the de Sitter manifold which is due to Dirac himself [5]. Let us consider the Clifford anti-commutation relations

$$
\gamma^{\alpha} \gamma^{\beta}+\gamma^{\beta} \gamma^{\alpha}=2 \eta^{\alpha \beta}
$$

relative to the three-dimensional Minkowski metric (3.4). The following choice

$$
\gamma^{0}=\left(\begin{array}{ll}
0 & 1 \\
1 & 0
\end{array}\right), \quad \gamma^{1}=\left(\begin{array}{cc}
0 & 1 \\
-1 & 0
\end{array}\right), \quad \gamma^{2}=\left(\begin{array}{cc}
i & 0 \\
0 & -i
\end{array}\right)
$$

provides a realisation of the above relations. The generators $L_{\alpha \beta}$ of the Lorentz group $S O_{0}(1,2)$ are given by

$$
L_{\alpha \beta}=M_{\alpha \beta}+S_{\alpha \beta},
$$

where $M_{\alpha \beta}=-i\left(X_{\alpha} \partial_{\beta}-X_{\beta} \partial_{\alpha}\right)$ and $S_{\alpha \beta}=-\frac{i}{4}\left[\gamma_{\alpha}, \gamma_{\beta}\right]$ are respectively the 'orbital' and the 'spinorial' parts of $L_{\alpha \beta}$. In the coordinates (3.5) the orbital components

$$
\begin{aligned}
& M_{01}=-i r \cos t \sin \theta \partial_{t}-i r \sin t \cos \theta \partial_{\theta} \\
& M_{02}=-i r \cos t \cos \theta \partial_{t}+i r \sin t \sin \theta \partial_{\theta} \\
& M_{12}=-i r \partial_{\theta}
\end{aligned}
$$


obviously do not include derivatives w.r.t. the radial variable $r$. The differential operators $L_{\alpha \beta}$ are therefore tangential to the de Sitter hyperboloid (3.3) and have a well defined action on functions and spinors defined just on it.

In the case under study $(s=1 / 2)$ the first Casimir operator takes the form ${ }^{1}$

$$
Q_{1}=-\frac{1}{2} L^{\alpha \beta} L_{\alpha \beta}=\left(\frac{1}{2} \gamma_{\alpha} \gamma_{\beta} M^{\alpha \beta}\right)^{2}+i \gamma_{\alpha} \gamma_{\beta} M^{\alpha \beta}-\frac{3}{4} .
$$

and its eigenvalues $\frac{1}{4}+\nu^{2}$ are parametrized by a nonzero real number $\nu$ as described in Bargmann's classic paper [6]. Since

$$
Q_{1}-\frac{1}{4}=\left(\frac{1}{2} \gamma_{\alpha} \gamma_{\beta} M^{\alpha \beta}+i\right)^{2}
$$

following Dirac [5] we may introduce another first order spinorial equation:

$$
(i D+i+\nu) \Psi=0
$$

where we have set

$$
i D=\frac{1}{2} \gamma_{\alpha} \gamma_{\beta} M^{\alpha \beta}
$$

\subsection{Spinorial plane waves}

The starting observation for solving eq. (4.8) is that the two-dimensional de Sitter d'Alembert's operator can be factorized as follows:

$$
\left(\begin{array}{cc}
\square & 0 \\
0 & \square
\end{array}\right)=\cos ^{2} t\left(\begin{array}{cc}
\partial_{t}^{2}-\partial_{\theta}^{2} & 0 \\
0 & \partial_{t}^{2}-\partial_{\theta}^{2}
\end{array}\right)=-i D(i D+i) .
$$

This relation implies that

$$
(i D+i+\nu)(-i D+\nu) \Psi=\left(\square+\nu^{2}+i \nu\right) \Psi .
$$

Given a two-component solution of the scalar de Sitter Klein-Gordon equation

$$
\left(\square+\nu^{2}+i \nu\right) \Psi=0
$$

we may construct a solution of eq. (4.8) simply by applying the operator $(-i D+\nu)$ to it [17]. Let us recall how to find a suitable basis of scalar solutions for the de Sitter KleinGordon equation $[14,15]$. Given a nonzero light-like vector $\xi \in M_{3}$ and a complex number $\lambda \in \mathbf{C}$ we construct the homogeneous function

$$
Z \mapsto(Z \cdot \xi)^{\lambda}
$$

${ }^{1}$ Eq. (4.6) follows from the identities

$$
\begin{aligned}
\frac{1}{2} M^{\alpha \beta} M_{\alpha \beta} & =-\left(\frac{1}{2} \gamma_{\alpha} \gamma_{\beta} M^{\alpha \beta}\right)^{2}-\frac{i}{2} \gamma_{\alpha} \gamma_{\beta} M^{\alpha \beta}, \\
M^{\alpha \beta} S_{\alpha \beta} & =-\frac{i}{2} \gamma_{\alpha} \gamma_{\beta} M^{\alpha \beta}, \quad \frac{1}{2} S^{\alpha \beta} S_{\alpha \beta}=\frac{3}{4} .
\end{aligned}
$$


where $Z$ belongs to the complex ambient Minkowski spacetime; this function is holomorphic in the tubes $T_{ \pm}=M_{3} \pm i V^{+}$, where $V^{+}$is the future cone of the origin, and satisfies the massless Klein-Gordon equation there. When restricted to the de Sitter universe it is holomorphic in the tuboids $\mathcal{T}_{ \pm}$obtained by interesting the ambient spacetime tubes $T_{ \pm}$ with the complex de Sitter manifold $d S_{2}^{c}$, and satisfies the massive (complex) Klein-Gordon equation:

$$
(\square-\lambda(\lambda+1))(Z \cdot \xi)^{\lambda}=0 .
$$

The parameter $\lambda$ is here unrestricted, i.e. we consider a complex squared masses $m_{\lambda}^{2}=$ $-\lambda(\lambda+1)$. The boundary values

$$
(X \cdot \xi)_{ \pm}^{\lambda}=\lim _{Z \in \mathcal{T}_{ \pm}, Z \rightarrow X}(Z \cdot \xi)^{\lambda},
$$

are homogeneous distributions of degree $\lambda$ on $M_{3}$, and their restrictions to $d S_{2}$, denoted with the same symbols, are solutions of the de Sitter Klein-Gordon equation

$$
\left(\square+m_{\lambda}^{2}\right)(X \cdot \xi)_{ \pm}^{\lambda}=0, \quad\left(\square+m_{\lambda}^{2}\right)(X \cdot \xi)_{ \pm}^{-1-\lambda}=0 .
$$

All these objects depend in a $\mathcal{C}^{\infty}$ way on $\xi$ and are entire in $\lambda$.

Coming back to the de Sitter-Dirac equation (4.8), spinorial plane waves can therefore be written in terms of scalar plane waves as follows [17]:

$$
(-i D+\nu)(X \cdot \xi)_{ \pm}^{\lambda} v(\xi)
$$

where $v=v(\xi)$ is a two-component spinor and the complex number $\lambda$ may take either of the following two values:

$$
\lambda_{1}=-i \nu, \quad \lambda_{2}=-1+i \nu .
$$

A straightforward calculation gives that

$$
-i D(X \cdot \xi)_{ \pm}^{\lambda} v(\xi)=i \lambda(X \cdot \xi)_{ \pm}^{\lambda-1} X w(\xi)-i \lambda(X \cdot \xi)_{ \pm}^{\lambda} v(\xi)
$$

where as usual $\not X=\gamma^{\alpha} X_{\alpha}$ and we set $w(\xi)=\$ v(\xi)$. The spinor $w(\xi)$ satisfies the condition $\$ w(\xi)=0$. In general, let us consider the linear equation

$$
\$ u(\xi)=0 .
$$

For any given lightlike vector $\xi$ the unique solution (apart from normalisation) of the above equation is

$$
u(\xi)=\frac{1}{\sqrt{2\left(\xi^{0}-\xi^{1}\right)}}\left(\begin{array}{c}
\xi^{0}-\xi^{1} \\
i \xi_{2}
\end{array}\right)=\frac{1}{\sqrt{2}}\left(\begin{array}{c}
\sqrt{\xi^{0}-\xi^{1}} \\
i \sqrt{\xi^{0}+\xi^{1}}
\end{array}\right)
$$

Interestingly, this expression coincides with Cartan's original definition of a spinor, as described in his classic book ([13] - Chapter 3). Defining the adjoint spinor in the usual way

$$
\bar{u}(\xi)=u^{\dagger}(\xi) \gamma^{0}
$$


there follows the completeness relation

$$
u(\xi) \otimes \bar{u}(\xi)=\frac{1}{2} \not \xi .
$$

Let us suppose that $v(\xi)$ itself solves eq. (4.19) and therefore that it has the form given in eq. (4.20). We obtain the following (complete, unnormalised) set of spinorial plane wave solutions, labeled by the wave vector $\xi$ and the mass parameter $\nu$ :

$$
(X \cdot \xi)_{ \pm}^{-1+i \nu} u(\xi) \text {. }
$$

where $u$ is the spinor given in eq. (4.20). It is now possible to build the two-point function of the quantum Dirac field $\Psi_{\nu}$ by superposing spinorial plane waves having the correct analyticity properties; these analyticity properties are there to replace of a true global spectral condition which is absent in de Sitter Quantum Field Theory [14-17]:

$$
\left(\Omega, \Psi_{\nu}\left(X_{1}\right) \bar{\Psi}_{\nu}\left(X_{2}\right) \Omega\right)=W_{\nu}\left(X_{1}, X_{2}\right)=\frac{c_{\nu}}{2} \int_{\gamma}\left(X_{1} \cdot \xi\right)_{-}^{-1+i \nu}\left(X_{2} \cdot \xi\right)_{+}^{-1-i \nu} \sharp d \mu_{\gamma}(\xi)
$$

and the canonical anticommutation relations fix the value of the constant:

$$
c_{\nu}=\frac{\Gamma(1-i \nu) \Gamma(1+i \nu)}{(2 \pi)^{2} e^{\pi \nu}} .
$$

Since

$$
X(-i D+\nu)(X \cdot \xi)^{-i \nu}=-\nu(X \cdot \xi)^{-1-i \nu} \not
$$

we get (in the complex analyticity domain $\mathcal{T}_{-} \times \mathcal{T}_{+}$)

$$
W_{\nu}\left(Z_{1}, Z_{2}\right)=-\frac{c_{\nu}}{2 \nu} \boldsymbol{Z}_{2}\left(-i D_{2}+\nu\right) \int_{\gamma}\left(Z_{1} \cdot \xi\right)_{-}^{-1+i \nu}\left(Z_{2} \cdot \xi\right)_{+}^{-i \nu} d \mu_{\gamma}(\xi)
$$

The integral at the r.h.s. is a hypergeometric function of the invariant scalar product $\zeta=Z_{1} \cdot Z_{2}$ (see appendix $\mathrm{B}$ ) and all the spinorial content is carried by the operator acting on it:

$$
W_{\nu}\left(Z_{1}, Z_{2}\right)=\frac{\Gamma(1-i \nu) \Gamma(i \nu)}{4 \pi} \mathbb{Z}_{2}\left(-i D_{2}+\nu\right) F\left(i \nu, 1-i \nu ; 1 ; \frac{1-\zeta}{2}\right)
$$

Taking the massless limit $\nu \rightarrow 0$ we get

$$
\begin{aligned}
W_{0}\left(Z_{1}, Z_{2}\right) & =-\frac{i}{4 \pi} \mathbb{Z}_{2}+\frac{1}{4 \pi} \mathbb{Z}_{2}\left(-i D_{2}\right) \sum_{n=1}^{\infty} \frac{1}{n}\left(\frac{1-\zeta}{2}\right)^{n}= \\
& =-\frac{i}{4 \pi} \mathbb{Z}_{2}+\frac{1}{4 \pi} \mathbb{Z}_{2}\left(-i D_{2}\right) \log \left(\frac{1+\zeta}{2}\right)=-\frac{i}{2 \pi} \frac{\left(\boldsymbol{Z}_{1}-\boldsymbol{Z}_{2}\right)}{\left(Z_{1}-Z_{2}\right)^{2}} .
\end{aligned}
$$

As a consequence of the above chain of identities we obtain a nice integral representation of the two-point function of the massless field $\Psi_{0}$ :

$$
\left(\Omega, \Psi_{0}\left(Z_{1}\right) \bar{\Psi}_{0}\left(Z_{2}\right) \Omega\right)=\frac{1}{2 \pi i} \frac{\left(Z_{1}-Z_{2}\right)}{\left(Z_{1}-Z_{2}\right)^{2}}=\frac{1}{8 \pi^{2}} \int_{\gamma}\left(Z_{1} \cdot \xi\right)^{-1}\left(Z_{2} \cdot \xi\right)^{-1} \Varangle d \mu_{\gamma}(\xi)
$$

valid for $Z_{1} \in \mathcal{T}^{-}$and $Z_{2} \in \mathcal{T}^{+}$. 


\section{Spin group and de Sitter covariance}

Let us discuss now the de Sitter covariance of the Dirac field. In our context the spin group (i.e. the double covering of $S O_{0}(1,2)$ ) is most usefully realized as the matrix group

$$
\operatorname{Sp}(1,2)=\left\{g \in \mathrm{SL}(2, C): \gamma^{0} g^{\dagger} \gamma^{0}=g^{-1}\right\} .
$$

An element of $\operatorname{Sp}(1,2)$ may be parametrised in terms of four real numbers $a, b, c, d$ subject to the condition $a d+b c=1$ as follows:

$$
g=\left(\begin{array}{cc}
a & i b \\
i c & d
\end{array}\right) .
$$

As a subgroup of $\operatorname{SL}(2, C)$, the group $\operatorname{Sp}(1,2)$ is conjugated to $\operatorname{SL}(2, R)$ : the following element of $\mathrm{SL}(2, C)$

$$
h=\left(\begin{array}{cc}
e^{\frac{i \pi}{4}} & 0 \\
0 & e^{-\frac{i \pi}{4}}
\end{array}\right)
$$

does the job

$$
h g h^{-1}=\left(\begin{array}{cc}
a & -b \\
c & d
\end{array}\right) .
$$

The group $\operatorname{Sp}(1,2)$ acts on the de Sitter manifold by similarity transformations:

$$
X^{\prime}=g X g^{-1} \text {. }
$$

The covering projection $g \rightarrow \Lambda(g)$ of $\operatorname{Sp}(1,2)$ onto $S O_{0}(1,2)$ coherent with the above action is expressed as follows:

$$
g \rightarrow \Lambda(g)_{\beta}^{\alpha}=\frac{1}{2} \operatorname{tr}\left(\gamma^{\alpha} g \gamma_{\beta} g^{-1}\right) .
$$

$\Lambda(g)$ is the (real) Lorentz transformation that directly relates $X^{\prime}$ and $X$

$$
g X g^{-1}=\Lambda(g) X \text {. }
$$

The transformation law for spinors under the action of $\operatorname{Sp}(1,2)$ is the standard one:

$$
\Psi^{\prime}(X)=g \Psi\left(\Lambda^{-1}(g) X\right), \quad \bar{\Psi}^{\prime}(X)=\bar{\Psi}\left(\Lambda^{-1}(g) z\right) g^{-1} .
$$

The covariance of the two-point function (4.24) under the transformation (5.8) can now be easily shown. In the massless case this is obvious

$$
g W_{0}\left(Z_{1}, Z_{2}\right) g^{-1}=\frac{1}{2 \pi i} \frac{g Z_{1} g^{-1}-g Z_{2} g^{-1}}{\left(Z_{1}-Z_{2}\right)^{2}}=\frac{1}{2 \pi i} \frac{\Lambda(g) Z_{1}-\Lambda(g) Z_{2}}{\left(Z_{1}-Z_{2}\right)^{2}} .
$$

In the general case, for any $g \in \operatorname{Sp}(1,2)$ there holds the following chain of equalities:

$$
\begin{aligned}
& g W_{\nu}\left(\Lambda^{-1}(g) X_{1}, \Lambda^{-1}(g) X_{2}\right) g^{-1}= \\
& =\frac{1}{2} c_{\nu} \int_{\Gamma}\left(\Lambda^{-1}(g) X_{1} \cdot \xi\right)^{-1+i \nu}\left(\Lambda^{-1}(g) X_{2} \cdot \xi\right)^{-1-i \nu} g \not g^{-1} d \mu(\xi) \\
& =\frac{1}{2} c_{\nu} \int_{\Gamma}\left(X_{1} \cdot \Lambda(g) \xi\right)^{-1+i \nu}\left(X_{2} \cdot \Lambda(g) \xi\right)^{-1-i \nu} \gamma^{\alpha}(\Lambda(g) \xi)_{\alpha} d \mu(\xi) \\
& =W_{\nu}\left(X_{1}, X_{2}\right)
\end{aligned}
$$

The last step is a consequence of the Stokes theorem exactly as in the scalar case [15]. 


\section{The symmetric space $\operatorname{Sp}(1,2) / A$ as the double covering of the two- dimensional de Sitter spacetime.}

In the following we need to dwell a little longer on the group $\operatorname{Sp}(1,2)$ and examine one of its coset spaces. The starting point is the Iwasawa decomposition $K N A$ of $\operatorname{Sp}(1,2)$ :

$$
g=k(\zeta) n(\lambda) a(\chi)=\left(\begin{array}{cc}
\cos \frac{\zeta}{2} & i \sin \frac{\zeta}{2} \\
i \sin \frac{\zeta}{2} & \cos \frac{\zeta}{2}
\end{array}\right)\left(\begin{array}{cc}
1 & i \lambda \\
0 & 1
\end{array}\right)\left(\begin{array}{cc}
e^{\frac{\chi}{2}} & 0 \\
0 & e^{-\frac{\chi}{2}}
\end{array}\right)
$$

the parameters $\zeta, \lambda$ and $\chi$ are related to $a, b, c$ and $d$ by the following relations:

$$
\cos \frac{\zeta}{2}=\frac{a}{\sqrt{a^{2}+c^{2}}}, \quad \sin \frac{\zeta}{2}=\frac{c}{\sqrt{a^{2}+c^{2}}}, \quad \lambda=a b-c d, \quad e^{\frac{\chi}{2}}=\sqrt{a^{2}+c^{2}}
$$

where $0 \leq \zeta<4 \pi$ and $\lambda$ and $\chi$ are real. Note that $a$ and $c$ cannot be both 0 since $a d+b c=1$. The above Iwasawa decomposition provides a natural parametrization of the coset space $\operatorname{Sp}(1,2) / A$

$$
\tilde{X}(\lambda, \zeta)=k(\zeta) n(\lambda)=\left(\begin{array}{cc}
\cos \frac{\zeta}{2} & i \lambda \cos \frac{\zeta}{2}+i \sin \frac{\zeta}{2} \\
i \sin \frac{\zeta}{2} & \cos \frac{\zeta}{2}-\lambda \sin \frac{\zeta}{2}
\end{array}\right)
$$

which is seen to be topologically a cylinder. The coset space $\operatorname{Sp}(1,2) / A$ is a symmetric space. The group $\operatorname{Sp}(1,2)$ acts on the coset space by left multiplication:

$$
g \tilde{X}(\lambda, \zeta) \rightarrow \tilde{X}\left(\lambda^{\prime}, \zeta^{\prime}\right)
$$

It is useful to describe the action of the subgroups separately. The case of a rotation $k(\alpha) \in K$ is of course the easiest one, it amounts simply to a shift of the angle $\zeta$ :

$$
\lambda^{\prime}(\alpha)=\lambda, \quad \zeta^{\prime}(\alpha)=\zeta+\alpha
$$

The two other subgroups give rise to slightly more involved transformation rules of the parameters $(\lambda, \zeta)$; an element $a(\kappa)$ of the abelian subgroup $A$ gives

$$
\left\{\begin{array}{l}
\lambda^{\prime}(\kappa)=\lambda \cosh \kappa+\sinh \kappa(\lambda \cos \zeta+\sin \zeta), \\
\cot \frac{\zeta^{\prime}(\kappa)}{2}=e^{\kappa} \cot \frac{\zeta}{2}
\end{array}\right.
$$

An element $n(\mu) \in N$ gives

$$
\left\{\begin{array}{l}
\lambda^{\prime}(\mu)=\lambda\left(1+\frac{1}{2} \mu^{2}\right)-\mu\left(\lambda+\frac{\mu}{2}\right) \sin \zeta+\mu\left(1-\frac{1}{2} \lambda \mu\right) \cos \zeta \\
\cot \frac{\zeta^{\prime}(\mu)}{2}=\cot \frac{\zeta}{2}-\mu .
\end{array}\right.
$$

The Maureer-Cartan form $d g g^{-1}$ gives to the symmetric space $\operatorname{Sp}(1,2) / A$ a natural Lorentzian metric that may be constructed as follows (see e.g. [18]). There exists a inner automorphism of $\operatorname{Sp}(1,2)$

$$
g \rightarrow \mu(g)=-\gamma^{2} g \gamma^{2}
$$


that leaves invariant the elements of the subgroup $A$. It may be used to construct a map from the coset space $\operatorname{Sp}(1,2) / A$ into the group $\operatorname{Sp}(1,2)$ :

$$
g(\tilde{X})=g \mu(g)^{-1}=-\tilde{X} \gamma^{2} \tilde{X}^{-1} \gamma^{2} .
$$

This map in turn allows to introduce a left invariant Lorentzian metric on the coset space as follows:

$$
d s^{2}=\frac{1}{2} \operatorname{Tr}\left(d g g^{-1}\right)^{2}=-2 d \lambda d \zeta-\left(\lambda^{2}+1\right) d \zeta^{2}
$$

One may verify directly that:

1. The metric (6.10) is invariant under the transformations (6.5), (6.6) and (6.7).

2. The curvature is constant $(R=-2)$ and the Ricci tensor is proportional to the metric:

$$
R_{\mu \nu}-\frac{1}{2} R g_{\mu \nu}=R_{\mu \nu}+g_{\mu \nu}=0
$$

3. The map $p: \operatorname{Sp}(1,2) / A \rightarrow d S_{2}$

$$
p: \tilde{X}(\lambda, \zeta) \rightarrow X(\lambda, \zeta)=\left\{\begin{array}{l}
X^{0}=-\lambda \\
X^{1}=\lambda \cos \zeta+\sin \zeta \\
X^{2}=\cos \zeta-\lambda \sin \zeta
\end{array}\right.
$$

is a covering map.

4. Restricting the ambient spacetime metric (3.4) to the de Sitter manifold with the coordinates (6.12) gives again

$$
d s^{2}=\left.\left(d X^{0^{2}}-d X^{1^{2}}-d X^{2^{2}}\right)\right|_{d S_{2}}=-2 d \lambda d \zeta-\left(\lambda^{2}+1\right) d \zeta^{2}
$$

In conclusion: the symmetric space $\operatorname{Sp}(1,2) / A=\widetilde{d S_{2}}$ may be identified with the double covering of the two-dimensional de Sitter universe. The spin group $\operatorname{Sp}(1,2)$ acts directly on the covering space $\widetilde{d S_{2}}$ as a group of spacetime transformations:

$$
\tilde{X} \rightarrow g \tilde{X}
$$

We were not able to find the above identification in the (enormous) literature on the group $\mathrm{SL}(2, R)$.

\section{$7 \quad$ Field dressing $\grave{a}$ la Gürsey and Lee}

Since there are two apparently distinct Dirac's equations on the de Sitter manifold, namely equation (3.11) and equation (4.8), it is natural to ask whether there is a relation between them and what it is. In four dimensions, this question has been raised first by Gürsey and Lee [7] and they provided a way to build a bridge between the two equations. 
The two-dimensional case is more tricky (also more interesting) because of its topological peculiarities. Following [7] let us introduce three matrices

$$
\beta^{\alpha} \equiv\left(\frac{\partial Y^{\alpha}}{\partial X^{\beta}}\right) \gamma^{\beta}
$$

where $Y^{0}=t, Y^{1}=\theta$ and $Y^{2}=r$ are the coordinates (3.5). In these coordinates the Minkowski metric of the ambient spacetime $M_{3}$ is written

$$
d s^{2}=\frac{r^{2}}{\cos ^{2} t}\left(d t^{2}-d \theta^{2}\right)-d r^{2}=r^{2} g_{i j} d Y^{i} d Y^{j}-d r^{2} .
$$

Restricting to $r=1$ we find again the de Sitter metric and the following relations:

$$
\begin{aligned}
\left\{\beta^{i}, \beta^{j}\right\} & =\left\{\alpha^{i}, \alpha^{j}\right\}=g^{i j}, \quad i, j=0,1, \\
\beta^{2} & =-\not X, \quad\left\{\beta^{i}, \beta^{2}\right\}=0 .
\end{aligned}
$$

Eq. (7.3) implies the existence of a matrix $S$ such that

$$
\alpha^{i}=S \beta^{i} S^{-1} .
$$

A particularly convenient choice - see the discussion below - is the following particular element $S(t, \theta)$ of the spin group $\operatorname{Sp}(1,2)$ :

$$
S(t, \theta)=\frac{1}{\sqrt{\cos t}}\left(\begin{array}{cc}
\cos \frac{t-\theta}{2} & i \sin \frac{t-\theta}{2} \\
-i \sin \frac{t+\theta}{2} & \cos \frac{t+\theta}{2}
\end{array}\right),
$$

but any other matrix of the form $S_{f}=f(t, \theta) S$, where $f(t, \theta)$ is an arbitrary function on the double-covering of the de Sitter spacetime $\widetilde{d S_{2}}$ is also permissible.

Given a solution $\Psi$ of the Dirac eq. (4.8) the dressed spinor

$$
\phi(t, \theta)=\frac{1}{\sqrt{2}} f(t, \theta) S(t, \theta)(1-X) \Psi(t, \theta)
$$

solves the equation

$$
i \alpha^{t}\left(\partial_{t}+\Gamma_{t}\right) \phi+i \alpha^{\theta}\left(\partial_{\theta}+\Gamma_{\theta}\right) \phi-i \alpha^{i}\left(\partial_{i} \ln f\right) \phi-\nu \phi=0
$$

(see appendix A). Clearly, the arbitrary function $f(t, \theta)$ can be reabsorbed by a gauge transformation. We may therefore $\operatorname{set}^{2} f=1$ in eq. (7.7) and observe the coincidence of eq. (7.8) with the covariant Dirac (Fock-Ivanenko) equation (3.12).

The matrix $S$ is anti-periodic $S(t, \theta+2 \pi)=-S(t, \theta)$ and therefore well-defined only on the double covering of the de Sitter hyperboloid. The map $(t, \theta) \rightarrow S(t, \theta)$ is thus a map from the double covering of the de Sitter spacetime $\widetilde{d S_{2}}$ with values in the spin group $\mathrm{Sp}(1,2)$ (see eq. (5.1)); when acting on a spinor field it changes its periodicity: periodic (R) fields become anti-periodic (NS) and viceversa.

\footnotetext{
${ }^{2}$ If the function $f$ is also anti-periodic, i.e. if $f(t, \theta+2 \pi)=-f(t, \theta)$ the matrix $S_{f}$ is well defined on the de Sitter hyperboloid $d S_{2}$ itself. However in this case we cannot solve the Dirac-Fock-Ivanenko equation with zero potential.
} 
The group element $S(t, \theta)=S(\tilde{X}(t, \theta))=S(\tilde{X})$ has a very simple geometrical interpretation that is made clear by examining the Lorentz transformation associated to it through the projection (5.6):

$$
\Lambda(S(t, \theta))=\left(\begin{array}{ccc}
\sec t & -\sin \theta \tan t & -\cos \theta \tan t \\
0 & \cos \theta & -\sin \theta \\
-\tan t & \sec t \sin \theta & \cos \theta \sec t
\end{array}\right)
$$

so that

$$
\Lambda(S(t, \theta)) X(t, \theta)=X(0,0)=\left(\begin{array}{l}
0 \\
0 \\
1
\end{array}\right)
$$

All the above features are not present in the original construction by Gürsey and Lee which was relative to the four-dimensional case.

\section{Cocyclic covariance of the de Sitter Dirac-Fock-Ivanenko field}

Let us apply the map (7.7) to the field $\Psi_{\nu}$ defined in eq. (4.24) and get a quantum field $\phi_{\nu}$ solving the standard Dirac (Fock-Ivanenko) equation. The field $\phi_{\nu}$ has the NS antiperiodicity and therefore is well-defined only on the manifold $\widetilde{d S_{2}}$. Eq. (5.8) tells us how the field $\phi_{\nu}$ is transformed by the action of the de Sitter group:

$$
\phi^{\prime}(\tilde{X})=\Sigma(g, \tilde{X}) \phi\left(g^{-1} \tilde{X}\right),
$$

where the matrix

$$
\Sigma(g, \tilde{X})=S(\tilde{X}) g S\left(g^{-1} \tilde{X}\right)^{-1}
$$

is also an element of the spin group $\operatorname{Sp}(1,2)$ depending on the point $\tilde{X} \in \widetilde{d S_{2}}$ and the group element $g$; one immediately verifies that $\Sigma(g, \tilde{X})$ is a nontrivial cocyle of $\operatorname{Sp}(1,2)$ :

$$
\Sigma\left(g_{1}, \tilde{X}\right) \Sigma\left(g_{2}, g_{1}^{-1} \tilde{X}\right)=\Sigma\left(g_{1} g_{2}, \tilde{X}\right) .
$$

The de Sitter covariance of the de Sitter Dirac NS field $\phi_{\nu}$ is thus expressed by eq. (8.1). On the other hand there is no covariant Dirac field (in the above sense) in the Ramond sector. The following remarkable result play an important technical role in the construction of the de Sitter-Thirring model (presented in a companion paper):

For any $g$ in the spin group $\operatorname{Sp}(1,2)$ the cocycle $\Sigma(g, \tilde{X})$ is diagonal.

We give a proof of the above statement by explicitly exhibiting $\Sigma(g, \tilde{X})$ for the oneparameter subgroups of the Iwasawa decomposition (6.1). Let us first parametrize the matrix $S(\tilde{X})$ with the Iwasawa coordinates $(\lambda, \zeta)$ of the point $\tilde{X}$ :

$$
S(\lambda, \zeta)=\left(1+\lambda^{2}\right)^{\frac{1}{4}}\left(\begin{array}{cc}
\cos \left(\frac{\zeta}{2}+\arctan \lambda\right) & -i \sin \left(\frac{\zeta}{2}+\arctan \lambda\right) \\
-i \sin \frac{\zeta}{2} & \cos \frac{\zeta}{2}
\end{array}\right) .
$$


1) For every spatial rotation $g=k$ and every $\tilde{X} \in \widetilde{d S_{2}}$ the cocycle $\Sigma(k, \tilde{X})=1$.

2) For a transformation $a(\kappa)$ belonging to the abelian subgroup $A$ we have

$$
\Sigma(a(\kappa), \tilde{X})=\left(\begin{array}{cc}
e^{-\kappa / 2} \frac{\left(1+\lambda^{\prime 2}\right)^{\frac{1}{4}}}{\left(1+\lambda^{2}\right)^{\frac{1}{4}}} \sin \left(\frac{\zeta^{\prime}}{2}\right) & 0 \\
0 & \left.e^{\kappa / 2} \frac{\left(1+\lambda^{2}\right)^{\frac{1}{4}}}{\left(1+\lambda^{\prime 2}\right)^{\frac{1}{4}} \sin \left(\frac{\zeta}{2}\right)} \sin \frac{\zeta^{\prime}}{2}\right)
\end{array}\right)
$$

where

$$
\left\{\begin{array}{l}
\lambda^{\prime}=\lambda^{\prime}(-\kappa)=\lambda \cosh \kappa-\sinh \kappa(\lambda \cos \zeta+\sin \zeta) \\
\cot \frac{\zeta^{\prime}}{2}=\cot \frac{\zeta^{\prime}(-\kappa)}{2}=e^{-\kappa} \cot \frac{\zeta}{2}
\end{array}\right.
$$

3) For a transformation $n(\mu)$ belonging to the upper triangular subgroup $N$ we have

$$
\Sigma(n(\mu), \tilde{X})=\left(\begin{array}{cc}
\frac{\left(1+\lambda^{\prime 2}\right)^{\frac{1}{4}}}{\left(1+\lambda^{2}\right)^{\frac{1}{4}}} \sin \left(\frac{\zeta^{\prime}}{2}\right) & \sin \left(\frac{\zeta}{2}\right) \\
0 & \frac{\left(1+\lambda^{2}\right)^{\frac{1}{4}} \sin \left(\frac{\zeta}{2}\right)}{\left(1+\lambda^{\prime 2}\right)^{\frac{1}{4}} \sin \left(\frac{\zeta^{\prime}}{2}\right)}
\end{array}\right)
$$

where

$$
\left\{\begin{array}{l}
\lambda^{\prime}=\lambda^{\prime}(-\mu)=\frac{1}{2}\left(\mu(2 \lambda-\mu) \sin \zeta-\mu(\lambda \mu+2) \cos \zeta+\lambda\left(\mu^{2}+2\right)\right) \\
\cot \frac{\zeta^{\prime}}{2}=\cot \frac{\zeta^{\prime}(-\mu)}{2}=\cot \frac{\zeta}{2}+\mu
\end{array}\right.
$$

\section{Massless fields: from the the de Sitter manifold to the cylinder and back}

In this concluding section we examine the various incarnations of the massless Dirac field. As explained in appendix A, in the massless case the dressing is simpler (see eq. (A.18)):

$$
\psi(t, \theta)=\frac{1}{\sqrt{\cos t}} S(t, \theta) \Psi_{0}(t, \theta)
$$

The 1.h.s. has to be understood as a Dirac field on the double covering of the cylinder obtained from the massless field eq. (4.30). Computing the two-point function we get

$$
\frac{S(t, \theta)\left(\Omega, \Psi_{0}(t, \theta) \bar{\Psi}_{0}\left(t^{\prime}, \theta^{\prime}\right) \Omega\right) S\left(t^{\prime}, \theta^{\prime}\right)^{-1}}{\sqrt{\cos t} \sqrt{\cos t^{\prime}}}=-\frac{i}{4 \pi}\left(\begin{array}{cc}
0 & \frac{1}{\sin \left(\frac{1}{2}\left(u-u^{\prime}\right)\right)} \\
\frac{1}{\sin \left(\frac{1}{2}\left(v-v^{\prime}\right)\right)} & 0
\end{array}\right)
$$

The two-point function completely characterizes the field: the remarkable result is that by the above construction the covariant massless de Sitter-Dirac field (4.29) is precisely mapped into the Neveu-Schwarz-Dirac field on the cylinder

$$
\psi^{\mathrm{NS}}(t, \theta)=\frac{1}{\sqrt{\cos t}} S(t, \theta) \Psi_{0}(t, \theta)
$$


and viceversa

$$
\Psi_{0}(t, \theta)=\sqrt{\cos t} S(t, \theta)^{-1} \psi^{\mathrm{NS}}(t, \theta)
$$

It is also instructive to apply the inverse transform (9.4) to the two components of the field (2.13) separately:

$$
\Psi_{0, r(l)}(t, \theta)=\sqrt{\cos t} S(t, \theta)^{-1} \psi_{1(2)}^{\mathrm{NS}}(t, \theta) .
$$

We get in this way a splitting of the massless Dirac field $\Psi_{0}=\Psi_{0, r}+\Psi_{0, l}$ into its right and left moving parts (i.e. the parts depending only on the $u$ and $v$ variables):

$$
\left(\Omega, \Psi_{0, r(l)}(t, \theta) \bar{\Psi}_{0, r(l)}\left(t^{\prime}, \theta^{\prime}\right)\right\rangle=\frac{1}{4 \pi i}(1 \pm i X)(X-Y)^{-1}
$$

where

$$
\begin{gathered}
\frac{1}{4 \pi i}(1+i X)(X-Y)^{-1}=A\left(u, u^{\prime}\right)=\left(\begin{array}{cc}
\frac{\cos \frac{u}{2} \sin \frac{u^{\prime}}{2}}{4 \pi \sin \left(\frac{1}{2}\left(u-u^{\prime}\right)\right)} & \frac{i \cos \frac{u}{2} \cos \frac{u^{\prime}}{2}}{4 \pi \sin \left(\frac{1}{2}\left(u-u^{\prime}\right)\right)} \\
\frac{i \sin \frac{u}{2} \sin \frac{u^{\prime}}{2}}{4 \pi \sin \left(\frac{1}{2}\left(u-u^{\prime}\right)\right)} & -\frac{\sin \frac{u}{2} \cos \frac{u^{\prime}}{2}}{4 \pi \sin \left(\frac{1}{2}\left(u-u^{\prime}\right)\right)}
\end{array}\right) \\
\frac{1}{4 \pi i}(1-i X)(X-Y)^{-1}=B\left(v, v^{\prime}\right)=\left(\begin{array}{cc}
\frac{\sin \frac{v}{2} \cos \frac{v^{\prime}}{2}}{4 \pi \sin \left(\frac{1}{2}\left(v-v^{\prime}\right)\right)} & \frac{i \sin \frac{v}{2} \sin \frac{v^{\prime}}{2}}{4 \pi \sin \left(\frac{1}{2}\left(v-v^{\prime}\right)\right)} \\
\frac{i \cos \frac{v}{2} \cos \frac{v^{\prime}}{2}}{4 \pi \sin \left(\frac{1}{2}\left(v-v^{\prime}\right)\right)} & -\frac{\cos \frac{v}{2} \sin \frac{v^{\prime}}{2}}{4 \pi \sin \left(\frac{1}{2}\left(v-v^{\prime}\right)\right)}
\end{array}\right)
\end{gathered}
$$

These expression are useful in computing the image of the Ramond field (2.9) under the same transformation:

$$
\Psi_{0}^{\mathrm{R}}(t, \theta)=\sqrt{\cos t} S(t, \theta)^{-1} \psi^{\mathrm{R}}(t, \theta) .
$$

The field $\Psi_{0}^{\mathrm{R}}(t, \theta)$ is defined on the double covering of the de Sitter spacetime and solves the massless Dirac equation (4.8) there. Its two-point function is written in the simplest way as follows in terms of the left and right part of the covariant two-point function as follows:

$$
\left(\Omega, \Psi_{0}^{\mathrm{R}}(t, \theta) \bar{\psi}_{0}^{\mathrm{R}}\left(t^{\prime}, \theta^{\prime}\right) \Omega\right)=\cos \left(\frac{1}{2}\left(u-u^{\prime}\right)\right) A\left(u, u^{\prime}\right)+\cos \left(\frac{1}{2}\left(v-v^{\prime}\right)\right) B\left(v, v^{\prime}\right)
$$

On the other hand, expressing the above two-point function using the ambient space variables gives a very complicated expression, not particularly useful. The representation (9.10) allows to prove that the $\Psi_{0}^{\mathrm{R}}(t, \theta)$ is covariant under rotations; namely when $g=g\left(\theta_{0}\right)=\kappa\left(\theta_{0}\right)$ is a spatial rotation then

$$
g\left(\theta_{0}\right)\left\langle\Psi_{0}^{\mathrm{R}}(t, \theta) \bar{\psi}_{0}^{\mathrm{R}}\left(t^{\prime}, \theta^{\prime}\right)\right\rangle g\left(-\theta_{0}\right)=\left(\Omega, \Psi_{0}^{\mathrm{R}}\left(t, \theta+\theta_{0}\right) \bar{\psi}_{0}^{\mathrm{R}}\left(t^{\prime}, \theta^{\prime}+\theta_{0}\right) \Omega_{0}\right)
$$

On the other hand, the boosts are broken. 


\section{A Derivation of the Gursey and Lee's dressing}

The Dirac operator can be rewritten as follows

$$
i D=\frac{1}{2} \gamma^{\alpha} \gamma^{\beta} M_{\alpha \beta}=\not X P-(X P)
$$

where $P_{\alpha}=-i \partial_{\alpha}$ and $X \cdot P=(X P)$. Note that, while the operator $i D$ does not include derivatives w.r.t. the radial variable $r$ the operators at the r.h.s. separately do. The important on-shell identity for what follows is the anticommutator:

$$
\{i D, X\}=-2 i X .
$$

Let $\Psi$ be a solution of the Dirac equation (4.8) and define

$$
\chi=\frac{1}{\sqrt{2}}(1-\not) \Psi .
$$

Eq. (A.2) implies that

$$
[i D+i+\nu](1+\not X) \chi=i(1-\not X) \chi+\nu(1+\not X) \chi+(1-\not X) i D \chi=0
$$

By multiplying the left of the r.h.s. by the operator $(1-\not X)$ and taking into account that $\chi$ does not depend on the radial variable we get

$$
-i X \chi+\nu \chi-X i D \chi=-i \beta^{a} \partial_{a} \chi+i \beta^{2} \chi+\nu \chi=0 .
$$

The latter identity is known as the Gursey and Lee's equation. Let us now define

$$
\phi=S(t, \theta) \chi
$$

The spinor $\phi$ satisfies the following equation:

$$
-i \alpha^{a} \partial_{a} \phi-i \alpha^{a} S\left(\partial_{a} S^{-1}\right) \chi+i \gamma^{2} \phi+\nu \phi=0
$$

Using the identity

$$
\alpha^{t} \alpha_{\theta}+\alpha^{\theta} \alpha_{t}=-2 i \gamma^{2}
$$

we may rewrite it as follows:

$$
i \alpha^{t}\left(\partial_{t}-\left(\partial_{t} S\right) S^{-1}-\frac{i}{2} \alpha_{\theta}\right) \phi+i \alpha^{\theta}\left(\partial_{\theta}-\left(\partial_{\theta} S\right) S^{-1}-\frac{i}{2} \alpha_{t}\right) \phi-\nu \phi=0
$$

Finally, the identifications

$$
-\left(\partial_{t} S\right) S^{-1}-\frac{i}{2} \alpha_{\theta}=0=\Gamma_{t}, \quad-\left(\partial_{\theta} S\right) S^{-1}-\frac{i}{2} \alpha_{t}=\left(\begin{array}{cc}
-\frac{1}{2} \tan t & 0 \\
0 & \frac{1}{2} \tan t
\end{array}\right)=\Gamma_{\theta},
$$

show that eq. (A.9) coincides with the covariant Dirac (Fock-Ivanenko) equation (3.12). The above construction more or less coincide with the original proposal by Gursey and 
Lee. We may also proceed the other way around and learn something. Let again $\Psi$ be a solution of the Dirac equation (4.8) and define first

$$
\zeta=S(t, \theta) \Psi
$$

Then

$$
S[i D+i+\nu] S^{-1} \zeta=-\gamma^{2} S \not P S^{-1} \zeta+(i+\nu) \zeta=0
$$

or else

$$
S \not P S^{-1} \zeta+(i+\nu) \gamma^{2} \zeta=-i \alpha^{i}\left(\partial_{i}+\Gamma_{i}\right) \zeta+\nu \gamma^{2} \zeta=0
$$

where again we took into account the fact that $\zeta$ does not depend on $r$. By defining

$$
\zeta=\frac{1}{\sqrt{2}}\left(1-\gamma^{2}\right) \phi
$$

we get again

$$
\left(1+\gamma^{2}\right)\left[i \alpha^{i}\left(\partial_{i}+\Gamma_{i}\right) \phi-\nu \phi\right]=0
$$

This second step is unnecessary in the massless case. Summarising, given a solution of the Dirac equation (4.8) the spinor

$$
\phi(t, \theta)=\frac{1}{\sqrt{2}} S(t, \theta)(1-X) \Psi(t, \theta)=\frac{1}{\sqrt{2}}\left(1+\gamma^{2}\right) S(t, \theta) \Psi(t, \theta)
$$

solves the covariant Dirac (Fock-Ivanenko) equation (3.12) (with $m=\nu$ ). The inverse map is

$$
\Psi(t, \theta)=\frac{1}{\sqrt{2}}(1+\not X) S(t, \theta)^{-1} \phi(t, \theta)=\frac{1}{\sqrt{2}} S(t, \theta)^{-1}\left(1-\gamma^{2}\right) \phi(t, \theta)
$$

In the massless case the above maps reduce to

$$
\phi_{0}(t, \theta)=S(t, \theta) \Psi_{0}(t, \theta)
$$

Taking into account the conjugacy relation $\gamma^{0} S^{+} \gamma^{0}=S^{-1}$ (defining our realisation of $\operatorname{Sp}(1,2))$ we also have

$$
\bar{\phi}(t, \theta)=\phi^{+}(t, \theta) \gamma^{0}=\frac{1}{\sqrt{2}} \bar{\Psi}(t, \theta)(1+\not) S(t, \theta)^{-1}=\frac{1}{\sqrt{2}} \bar{\psi}(t, \theta) S(t, \theta)^{-1}\left(1-\gamma^{2}\right) .
$$

\section{B Maximally analytic vacua}

We summarise here a small part of the scalar harmonic analysis on the de Sitter space (see e.g. [15]) used in the text. The main result is the following: for $z \in \mathcal{T}_{-}$and $z^{\prime} \in \mathcal{T}_{+}$in $d$ spacetime dimensions one has

$$
\begin{aligned}
& \int_{S_{0}}(\xi \cdot z)^{1-d-\lambda}\left(\xi \cdot z^{\prime}\right)^{\lambda} d \xi=\int_{\gamma}(\xi \cdot z)^{1-d-\lambda}\left(\xi \cdot z^{\prime}\right)^{\lambda} \alpha(\xi)= \\
& =\frac{2 \pi^{\frac{d}{2}} e^{i \pi\left(\lambda+\frac{d-1}{2}\right)}}{\Gamma\left(\frac{d}{2}\right)} F\left(-\lambda, \lambda+d-1 ; \frac{d}{2} ; \frac{1-\zeta}{2}\right), \quad \zeta=z \cdot z^{\prime}, \quad \lambda \in \mathbf{C} .
\end{aligned}
$$


Here $\gamma$ is any smooth $(d-1)$-cycle homotopic to the unit sphere $S_{0}$ in $C_{+} \backslash\{0\}$ and $\alpha$ is the $(d-1)$-differential form obtained from the rotation invariant measure $d \xi$ on the sphere. Both sides of (B.1) are entire in $\lambda \in \mathbf{C}$, as well as the r.h.s. of (B.2). If $\lambda$ is not a pole of $\Gamma(-\lambda) \Gamma(\lambda+d-1), z \in \mathcal{T}_{-}, z^{\prime} \in \mathcal{T}_{+}$, we denote

$$
\begin{aligned}
w_{\lambda}\left(z \cdot z^{\prime}\right) & =c(\lambda) \int_{S_{0}}(\xi \cdot z)^{1-d-\lambda}\left(\xi \cdot z^{\prime}\right)^{\lambda} d \xi, \\
c(\lambda) & =\frac{\Gamma(-\lambda) \Gamma(\lambda+d-1) e^{-i \pi\left(\lambda+\frac{d-1}{2}\right)}}{2^{d+1} \pi^{d}} .
\end{aligned}
$$

Two equivalent expressions for $w_{\lambda}$ :

$$
\begin{aligned}
w_{\lambda}\left(z \cdot z^{\prime}\right) & =\frac{\Gamma(-\lambda) \Gamma(\lambda+d-1)}{(4 \pi)^{d / 2} \Gamma\left(\frac{d}{2}\right)} F\left(-\lambda, \lambda+d-1 ; \frac{d}{2} ; \frac{1-\zeta}{2}\right) \\
& =\frac{\Gamma(-\lambda) \Gamma(\lambda+d-1)}{2(2 \pi)^{d / 2}}\left(\zeta^{2}-1\right)^{-\frac{d-2}{4}} P_{\lambda+\frac{d-2}{2}}^{-\frac{d-2}{2}}(\zeta), \quad \zeta=z \cdot z^{\prime} .
\end{aligned}
$$

\section{Acknowledgments}

U.M thanks the Institut des Hautes Etudes Scientifiques (Bures-sur-Yvette) for its hospitality and support.

Open Access. This article is distributed under the terms of the Creative Commons Attribution License (CC-BY 4.0), which permits any use, distribution and reproduction in any medium, provided the original author(s) and source are credited.

\section{References}

[1] P. Di Francesco, P. Mathieu and D. Sénéchal, Conformal Field Theory, Springer, New York U.S.A. (1996).

[2] M. Green, J.H. Schwarz and E. Witten, Superstring Theory, Cambridge University Press, Cambridge U.K. (1987).

[3] J. Cohn, D. Friedan, Z.-a. Qiu and S.H. Shenker, Covariant Quantization of Supersymmetric String Theories: The Spinor Field of the Ramond-Neveu-Schwarz Model,

Nucl. Phys. B 278 (1986) 577 [inSPIRE].

[4] N.D. Birrell and P.C.W. Davies, Quantum fFields in Curved Space, Cambridge University Press, Cambridge U.K. (1984).

[5] P.A.M. Dirac, The Electron Wave Equation in De-Sitter Space, Annals Math. 36 (1935) 657 [INSPIRE].

[6] V. Bargmann, Irreducible unitary representations of the Lorentz group, Annals Math. 48 (1947) 568 [inSPIRE].

[7] F. Gursey and T.D. Lee, Spin 1/2 Wave Equation in de Sitter Space, Proc. Nat. Acad. Sci. 49 (1963) 179 [inSPIRE].

[8] C.J. Isham, Twisted Quantum Fields in a Curved Space-Time, Proc. Roy. Soc. Lond. A 362 (1978) 383. 
[9] C.J. Isham, Spinor Fields in Four-dimensional Space-time, Proc. Roy. Soc. Lond. A 364 (1978) 591 [InSPIRE].

[10] S.J. Avis and C.J. Isham, Lorentz Gauge Invariant Vacuum Functionals for Quantized Spinor Fields in Nonsimply Connected Space-times, Nucl. Phys. B 156 (1979) 441 [INSPIRE].

[11] D. Wolf and J. Zittartz, Physics of the Schwinger model, Z. Phys. B 59 (1985) 117.

[12] N.S. Manton, The Schwinger Model and Its Axial Anomaly, Annals Phys. 159 (1985) 220 [INSPIRE].

[13] É. Cartan, La Théorie des Spineurs, Hermann, Paris France (1938).

[14] J. Bros, U. Moschella and J.P. Gazeau, Quantum field theory in the de Sitter universe, Phys. Rev. Lett. 73 (1994) 1746 [INSPIRE].

[15] J. Bros and U. Moschella, Two point functions and quantum fields in de Sitter universe, Rev. Math. Phys. 8 (1996) 327 [gr-qc/9511019] [INSPIRE].

[16] J. Bros, H. Epstein and U. Moschella, Analyticity properties and thermal effects for general quantum field theory on de Sitter space-time, Commun. Math. Phys. 196 (1998) 535 [gr-qc/9801099] [INSPIRE].

[17] P. Bartesaghi, J.P. Gazeau, U. Moschella and M.V. Takook, Dirac fields and thermal effects in the de Sitter universe, Class. Quant. Grav. 18 (2001) 4373 [INSPIRE].

[18] H. Eichenherr and M. Forger, More about nonlinear $\sigma$-models on symmetric spaces, Nucl. Phys. B 164 (1980) 528 [Erratum ibid. B 282 (1987) 745] [InSPIRE]. 